\title{
QUINE: SOBRE REFERENCIA Y RELATIVIDAD
}

\section{María Inés Prono}

\section{La teoría de la significación traductiva}

$\mathrm{Al}$ comienzo del Capítulo 2 de su ya célebre Palabra y objeto, Quine presenta su no menos célebre tesis de la indeterminación de la traducción radical. Su formulación menos abstracta, según él mismo nos dice, adopta la siguiente forma:

“...es posible confeccionar manuales de traducción de una lengua a otra de diferentes modos, todos compatibles con la totalidad de las disposiciones verbales $y$, sin embargo, todos incompatibles unos con otros. Estos manuales diferirán en numerosos puntos: como traducción de una sentencia de un lenguaje darán sentencias del otro que no encontrarán entre sí en ninguna relación de equivalencia plausible, por laxa que esta sea". ${ }^{1}$

Uno de los aspectos importantes que hay que hacer notar, es que las disposiciones verbales de las que habla Quine deben ser disposiciones verbales observables actualmente, en otras palabras, se refiere a los comportamientos lingüísticos actuales de los hablantes. Dentro de un planteo behaviorista, toda conducta se

${ }^{1}$ Quine, W.V., Palabra y Objeto (1960), Barcelona, Labor, 1968, p. 41. En adelante PyO. 
entiende como una respuesta a un estímulo que, en este caso, debe ser también observable.

Considerada desde un punto de vista metodológico, la tesis (o hipótesis) de indeterminación de la traducción es, en algún sentido, una hipótesis empírica, por lo tanto, debe ser empíricamente contrastable; esto es, para sostenerla como hipótesis aceptable, se debe contar con evidencia observacional favorable. ${ }^{2}$ Por otra parte, no es casual que en el primer capítulo de PyO se dedique una sección a la consideración de la metodología y procedimientos habituales de las ciencias naturales: predicción, acuerdo con la observación, generalización, criterio de simplicidad y familiaridad de los principios. Así las cosas, la empresa de traducción será llevada a cabo por un científico empírico: el lingüista.

La centralidad que adopta èl problema de la traducción en los escritos de Quine depende enteramente del modo en el que plantea su teoría del significado. Es fácil advertir, que la centralidad mencionada radica en que a Quine le interesa averiguar si es posible aclarar o elucidar el concepto mismo de 'significado', en el sentido de encontrar razones que permitan decidir si es necesario y útil mantener una noción como ésta. Esta convicción lo lleva a plantear el problema de la traducción en términos de una relación de equivalencia que -aunque no define explícitamente- puede ser entendida como 'mismidad de significado', o lo que técnicamente se ha denominado 'sinonimia'. En «Dos Dogmas del Empirismo» Quine había puesto seriamente en duda esta última noción en rela-

\footnotetext{
${ }^{2}$ Como bien señala R. Orayen, "Una importante conclusión que se sigue de estas consideraciones es que para Quine, entonces, es posible obtener conocimiento legítimo sobre las disposiciones verbales mediante métodos experimentales inductivos similares a los de las ciencias físicas". Orayen, R., "Indeterminación de la traducción y epistemología naturalizada", en Análisis Filosófico, Buenos Aires, SADAF, 1990, p.105.
} 
ción al supuesto estatuto de aprioridad de los enunciados analíti$\cos .{ }^{3}$ La misma noción de sinonimia parece ser también la que está en juego al tratar el problema de la traducción, tal como se la plantea en Palabra y Objeto.

"Pues suponemos que la significación es lo que una sentencia tiene en común con su traducción; y traducción, en este estadio de nuestro estudio, se refiere sólo a correlación con estimulaciones no verbales". (PyO p. 45) resaltado mío

Una respuesta natural o intuitiva a la pregunta por la sinonimia consistiría en decir esto: una traducción concreta de una

${ }^{3}$ Las objeciones de Quine al concepto de sinonimia, expuestas en «Dos Dogmas del Empirismo», consisten en mostrar que el mismo no puede ser definido adecuadamente sino es apelando a otros términos semánticos relacionados con él, como por ej.: 'analítico', 'definición', 'regla semántica'. Por esta razón, sugiere que dichos términos son inapropiados, oscuros y hasta ininteligibles; con lo cual, su utilidad explicativa es escasa o nula, y sería más conveniente prescindir de ellos. Muchos han sido los críticos que observan que estos argumentos no son concluyentes para justificar el abandono de tales nociones. Cf. P. Grice \& P. Strawson. (1956) «In defense of a dogma», in E. Nagel \& R. Brandt (eds.), 1965. Meaning and Existence. Systematic Readings in Epistemology. New York: Harcourt, Brace \& World. En un trabajo muy claro, J.J. Acero hace notar que si bien estas objeciones son acertadas, sólo cuentan la mitad de la historia. Los argumentos semánticos de «Dos dogmas...» se complementan con otros expuestos en «Referencia y modalidad» en los que se analizan los problemas derivados de la aceptación de contextos referencialmente opacos y del empleo de la sustitución por identidad. Estos expedientes implicarían la eliminación de objetos tales como los individuos y las clases, que son imprescindibles en la ontología de los lenguajes científicos. Las ontologías intensionales son rechazadas por ser incompatibles con las extensionales; las primeras tienen efectos devastadores para el lenguaje científico. Cf. J.J. Acero «Quine y la analiticidad. (Una lectura de «Dos dogmas del empirismo»)». Seminarios de Filosofía, N6, 1993, Pontificia Universidad Católica de Chile, pp. 37-40. 
expresión de una lengua extranjera es correcta cuando, y sólo cuando, una y otra oración expresan la misma idea o idéntico significado. Pero éste es precisamente el concepto de sinonimia que Quine no acepta. Esta salida sería propia de una semántica acrítica e intuitiva que, desde el empirismo radical sostenido por Quine, debe ser abandonada pues no reporta ningún beneficio explicativo, dado que no cuenta con el mismo soporte evidencial que una teoría basada en las disposiciones a la conducta lingüística. Pero además, Quine dice rechazar la teoría de la copia o mito del museo, según las cuales las palabras serían rótulos o etiquetas adheridas a ciertas entidades (mentales o platónicas): los significados. Lo que se pretende rechazar, o lo que es necesario abandonar, es cierto principio de determinación del significado.

El concepto de significado que le resulte útil al lingüista puesto en funciones de traductor, será aquel en el que quede reflejada la disposición del hablante (nativo) a asentir o disentir de sus preguntas en presencia de los estímulos sensoriales oportunos. Y esto es a lo que Quine llama significación estimulativa.

\section{Significación estimulativa}

En orden a dotar al lingüista de un definición de significación que guíe su tarea y le permita mantenerse apegado a las conductas verbales manifiestas de los hablantes, Quine propone un "rudo concepto" de significación empírica que tenga como requisito el de ser técnicamente neutro.

"podemos empezar por definir la significación estimulativa afirmativa: ...Una estimulación s pertenece a la significación estimulativa afirmativa de una sentencia $S$ para un hablante dado sí y sólo sí 
hay una estimulación s' tal que si se ofreciera al hablante s', se le preguntara luego $S$, se le ofreciera s y luego se le preguntara $S$ de nuevo, discreparía la primera vez y asentiría la segunda”. (PyO p.45)

La definición de significación estimulativa negativa, procede del mismo modo, cambiando de lugar asentiría y discreparía. La significación estimulativa se definiría como el par ordenado de las dos. La definición más exacta de Quine considera otras circunstancias. A este concepto de significación se lo vincula con un momento $t$ y con un intervalo de tiempo, puesto que hay que admitir que el hablante puede cambiar su comportamiento linguiístico. Pero lo más llamativo del caso es ver cómo redefine estimulativamente ciertas nociones básicas de la semántica analítica tradicional. Por ejemplo: un enunciado será estimulativamente analítico para un hablante dado, si éste asentiría a este enunciado en respuesta a toda estimulación; un enunciado es estimulativamente contradictorio para un hablante si su significación estimulativa (afirmativa) es el conjunto vacío, esto es, si el hablante, ante la interrogación por él, discrepa en toda estimulación. Para un hablante, dos enunciados son estimulativamente sinónimos si para él tienen la misma significación estimulativa. Esta es la única manera de admitir nociones primitivamente intensionales dentro del programa empirista y naturalista de la significación.

Dos aclaraciones son necesarias. Por una parte, las estimulaciones hacen referencia a todas aquellas irradiaciones que impacten nuestros receptores sensoriales, y se conciben como escenas enteras, al modo guestáltico. Esto pone de manifiesto que lo que puede causar el asentimiento o disenso no son los objetos propiamente dichos, sino situaciones completas que tienen una extensión espacial, en la que es posible establecer una relación entre centro y periferia. (PyO p. 43-45) Por otra parte, las estimulaciones son aquello que provocaría el asentimiento o disenso, en el senti- 
do de que son lo que activa la disposición del hablante a responder afirmativa o negativamente. Y Quine aclara que provocar no es determinar. (PyO p. 43)

Una dificultad importante se presenta al considerar que el asentimiento o disenso a una oración particular puede estar condicionado o influenciado por cierta información que posee el hablante, y que excede los límites de la estimulación presente, la que es denominada información intrusiva o colateral. Para superar esta dificultad, habrá que encontrar cierto tipo de oraciones en las que sea posible aislar o separar lo que solamente corresponde a su significación estimulativa, independientemente de lo que provenga de otro tipo de conocimiento anterior. Las oraciones que podrán ser traducidas de un modo altamente seguro, son las que Quine denomina oraciones observacionales.

"Cuanto menos sensible es a las influencias de información lateral la significación estimulativa de una sentencia ocasional, tanto menos absurdo es concebir la significación estimulativa como significación de la misma. Las sentencias ocasionales cuyas significaciones estimulativas no varían por la influencia de información lateral pueden llamarse muy naturalmente sentencias observacionales, y puede decirse sin temor a contradicción que sus significaciones estimulativas dan plena cuenta de sus significaciones".(PyO p. 55)

Una caracterización más o menos exhaustiva de las oraciones observacionales parece descansar sobre las siguientes tesis:

i) su valor de verdad varía con las circunstancias que prevalecen en el momento de su expresión. En el lenguaje de Quine estas son oraciones ocasionales. 
ii) las circunstancias que prevalecen en el momento de su expresión deben ser observables intersubjetivamente para todos los testigos de la situación.

iii) los testigos deben ser capaces de apreciar que la observación con la cual se enfrentan es una tal que verifica la oración. ${ }^{4}$

Ahora bien, la observacionalidad de una sentencia no será una propiedad absoluta, sino una cuestión de grado. En este sentido se distinguirán dos tipos de oraciones observacionales: las fijas o eternas y las ocasionales. Las más directamente relacionadas con las situaciones de estimulación son las últimas, por lo cual su observacionalidad será muy alta, mientras que en el caso de las primeras su relación con estimulaciones es indirecta, lo cual implica que su valor de verdad es relativamente independiente de las circunstancias que rodean a la emisión de las mismas. Por el segundo y tercero de los rasgos mencionados, las oraciones observacionales adquieren un estatuto objetivo y de dominio público, puesto que requieren de asentimiento intersubjetivo por parte de todos los testigos de la situación que sean competentes en el manejo del lenguaje. Esto justifica la afirmación de Quine de que la observacionalidad es una noción social.(PyO p. 57)

Fuera de las oraciones observacionales, que son las que están o podrían estar conectadas en forma directa con estímulos sensoriales, la mayoría de nuestras oraciones, tanto en el lenguaje ordinario como en el científico, dependen de un plus de información almacenada por la experiencia pasada y por los usos lingüísticos. Esta información lateral o conocimiento idiosincrático no puede, más que de una manera arbitraria, separarse de aquello que es causado por la estimulación. Una distinción de estos com-

${ }^{4}$ Quine, W.V.O., "The Nature of Natural Knowledge", en Guttemplan, S. (ed) (1975) Mind and Language, Clarendon Press, Oxford, p. 72. (En adelante NNK) 
ponentes es ilusoria, ya que se contienen mutuamente «en una solución que no precipita nunca» (PyO p. 52). De manera que, desde esta perspectiva, el mayor número de nuestras afirmaciones son teóricas, en el sentido de que su relación con la observación es indirecta y está mediada por el entrelazamiento con otras sentencias (holismo).

Además de las oraciones observacionales, también tendrán una traducción segura las funciones veritativas como la conjunción, la disyunción y la negación. Este tipo de oraciones, tan distintas de las anteriores, puede traducirse ya que es posible definir las funciones en términos de asentimiento y disenso de oraciones.

Reconstruida metodológicamente, tendrá -por el momento- los siguientes pasos:

1) Observar y registrar los comportamientos lingüísticos.

2) Observar y registrar las circunstancias (escenas) en que se éstos se desarrollan.

3) Inferir/descubrir por procedimientos de tanteo interrogativo las expresiones de asentimiento y disenso.

4) Proponer hipotéticamente una traducción de una expresión de la lengua remota por una supuestamente equivalente expresión en el sentido antes apuntado de mismidad de significación estimulativa- en su lengua materna.

5) Este último procedimiento debe realizarse oración por oración.

Quine continúa su relato del experimento de traducción radical tratando de mostrar cómo el lingüista debe mantenerse apegado a las manifestaciones observables de las conductas verbales de los hablantes nativos. La prudencia del lingüista dura muy poco, debido a que se debaten en él fuerzas contrapuestas: por una parte, en el supuesto de que tenga éxito con sus primeros ensayos, esto 
incentivará su creatividad; y segundo, las anteriores restricciones le dejan muy poco margen de acción para poder cumplir con la tarea de confeccionar un manual. Un lingüista puntilloso no pasará más allá de traducir sentencias observacionales por otras sentencias observacionales. Pero esto no es lo que se espera de él. Se espera que proporcione instrucciones para poder producir sentencias en otro lenguaje, al modo de un discurso coherente acerca del mundo.

"Un lingüista puntilloso es un lingüista enjaulado. ...lo que se exige del lingüista como producto terminado y útil no es una mera lista de equivalencias sentencia por sentencia... Lo que se necesita es un manual de instrucciones para formular de manera aproximada cualquier sentencia de nuestro idioma en la lengua nativa, y viceversa". ${ }^{5}$

\section{Indeterminación y Referencia:}

Para superar estas limitaciones el lingüista se aventurará a segmentar las sentencias en sus partes componentes, componiendo así una lista de «palabras» o términos que pondrá en correspondencia con palabras de su lengua materna. A partir de aquí formula o, comienza a emplear el método de las 'hipótesis analíticas' ó hipótesis de traducción. La función de éstas hipótesis no es la de proporcionar más correlaciones de sentencias con sentencias o de palabras con palabras. Estas hipótesis tienen que ver, por ejemplo, con lo que el lingüista supone que pueda ser un término general en la lengua nativa. Y esto implica manejar el aparato de individuación, de identidad y de cuantificación de esa lengua, del

${ }^{5}$ Quine, W.V., "Hablando de objetos" en La relatividad ontológica y otros ensayos, Madrid, Tecnos, 1974, p. 15. 
mismo modo como en nuestra lengua cumplen esa función algunos otros elementos auxiliares de la referencia objetiva, como los artículos y pronombres, las terminaciones en plural, la cópula, el predicado de identidad, etc. Lo que a esta altura puede resultar evidente es que estos expedientes de la lengua indígena no pueden ser conocidos en base a los comportamientos lingüísticos de los hablantes nativos, sino que su conocimiento excede ampliamente lo que por estos procedimientos se pueda llegar a constatar.

“Todos los elementos de ese aparato son
interdependientes, y la noción misma de término es
tan local o particular, tan propia de nuestra cultura,
como dichos expedientes auxiliares. ...Las senten-
cias ocasionales y la significación estimulativa son
moneda universal; los términos y la referencia son
locales, propios de nuestro esquema conceptual. ...el
uso de una palabra como sentencia ocasional, por
muy determinado que sea, no fija la extensión de la
palabra en cuanto término”.(PyO p. 66)

¿Cuál es, entonces, la situación que queda planteada en relación a la empresa de traducción radical? Por una parte, la noción de significación estimulativa no es equivalente a la noción intuitiva y preteórica de «significación». La orientación naturalista de Quine sostiene su visión de que el material objetivo concerniente a la sinonimia de expresiones debe ser encontrado en la conducta observable de los hablantes y no en alguna otra entidad supuesta. Desafortunadamente, la conducta observable nunca será capaz de arbitrar una decisión definitiva entre manuales de traducción rivales. La significación estimulativa no agota nunca lo que intuitivamente se ha entendido por significación. Con lo cual, no hay ningún elemento objetivo que nos permita captar la significación total de una expresión cualquiera. No hay 'fact of the matter' para el significado de una expresión. 
En segundo lugar, la referencia tampoco puede ser determinada, ya que nada nos autoriza a ello partiendo desde la constatación de las disposiciones a la conducta lingüística observable. Como se indicó antes, el aparato referencial de una lengua pertenece a su estructura abstracta; en el caso de nuestra lengua, son pautas de objetivación que vienen dadas principalmente por la identidad y la cuantificación. Estas pautas son locales, propias de cada lengua, y suponer que los miembros de comunidades lingüísticas remotas poseen pautas de objetivación similares o idénticas a las nuestras es una conjetura que no está apoyada por ninguna evidencia disponible. Por lo tanto, la referencia es inescrutable. No hay 'fact of the matter' para la referencia de una expresión.

Ahora bien, el conocimiento de las pautas de objetivación sólo puede inferirse por el recurso a las 'hipótesis analíticas'. La especificación de la referencia reposará siempre sobre ellas. Estas hipótesis proveen las bases para el análisis de la expresiones significativas en segmentos más pequeños. La diferencia entre este tipo de hipótesis y aquellas por las que se establecían las traducciones de oraciones observacionales, es que las primeras no serían hipótesis 'genuinas', esto es, no son hipótesis que puedan ser sometidas contrastación por los habituales procedimientos inductivos; para sostener este tipo de hipótesis, la evidencia observacional no cuenta más que de un modo indirecto. Son sustentadas por un tipo de evidencia diferente: la fluidez en el diálogo.

La indeterminación de la traducción atraviesa tanto a la intensión como a la extensión, o para usar términos fregeanos, la indeterminación afecta tanto al sentido como a la referencia de las expresiones. El rechazo explícito de Quine sobre toda noción intensional hace imposible el mantenimiento de la ecuación fregeana de que es el sentido el que determina a la referencia. 
Siguiendo a Roger Gibson Jr. la indeterminación de la traducción de Quine es de dos variedades y puede formularse como sigue: "una variedad es la indeterminación de la intensión o 'significado' (tomado en un sentido intuitivo) y puede afectar a una expresión considerada suficiente como para 'tener un significado'. La otra variedad es la indeterminación de la extensión, o indeterminación de la referencia y afecta a los términos. (Quine llama también a la última variedad inescrutabilidad de la referencia o inescrutabilidad de los términos)... Lo que ambas afirman, esencialmente, es que "significado" y referencia están indeterminados por los fundamentos conductuales y que investigar sobre la (posible) evidencia para un único 'significado' o referente es insensato". ${ }^{6}$

\section{Relatividad ontológica:}

De la inescrutabilidad de la referencia a la relatividad ontológica hay sólo un paso. Si las pautas de objetivación -esto es, los objetos acerca de los cuales alguien habla- son locales o propias de cada lengua, esto significa que la pregunta acerca de qué objetos hay sólo tiene sentido planteada como una cuestión interna a una lengua dada. Nuestro lenguaje ordinario, entendido como un marco conceptual, postula objetos.

"Decir cuáles son los objetos acerca de los cuales alguien habla no es más que decir cómo proponemos traducir sus términos a los nuestros: estamos en libertad de variar la decisión con una función de procuración. La traducción adoptada fija la referen-

${ }^{6}$ Gibson, R., "Translation, Physics and Facts of the matter" en The Philosophy of W.V. Quine, The Library of Living Philosophers. Volume XVIII. L. E. Hahn and P. A. Schilpp (eds.) Open Court, 1986, p. 146. (traducción mía) 
Quine: Sobre referencia y relatividad - 123

cia fluctuante de los términos ajenos sólo relativamente a la referencia fluctuante de nuestros propios términos, vinculando ambas". (TyC. p. 30)

El que hablemos de tales o cuales objetos como «reales» es una cuestión de uso del lenguaje y tiene que ver con las pautas objetivas mediante las cuales hemos aprendido el mismo y a través de las cuales, es significativo hablar de «realidad».

Podemos decir que nuestro lenguaje es nuestra más primitiva teoría de la naturaleza y que opera como marco conceptual de fondo de cualquier traducción; que la postulación de objetos externos obedece a nuestro «instinto o impulso» hacia la simplicidad, ya que simplificar nos permite manipular más sistemáticamente el flujo de nuestras estimulaciones sensoriales.

Entonces, simplificamos o reducimos la complejidad de nuestra corriente sensorial, postulando objetos externos dentro de las sentencias de nuestro lenguaje. Así se va construyendo nuestra ontología. Es preciso señalar que este procedimiento es análogo en el lenguaje ordinario y en el más especializado lenguaje científico.

En «Las cosas y su lugar en las teorías» Quine describe cómo construimos nuestra ontología. Resulta llamativo el modo en que va introduciendo los distintos objetos, o «poblando» la ontología (por ejemplo: con cuerpos físicos, números, clases, clases de clases, etc.). Este proceso de poblamiento se realiza de acuerdo a lo que necesitamos para elaborar nuestra teoría de la naturaleza. $^{7}$

La postulación de objetos externos es una función que se realiza en forma natural en el lenguaje ordinario y, en forma más consciente en el lenguaje científico. Desde un punto de vista

${ }^{7}$ Quine, W.V., «Las cosas y su lugar en las teorías», en Teorías y cosas. México, UNAM, 1986. En adelante TyC. 
cognoscitivo, sólo difieren en grado de antigüedad. ${ }^{8}$ Hablar de los objetos como reales pertenece al ámbito de lo hipotético, y el modo de pensar y hablar hipotéticos son rasgos específicos de la naturaleza humana que desempeñan una función importante para la supervivencia. En este sentido, no habrá mayor diferencia entre perros o sillas y átomos o moléculas. Así vistos los objetos físicos ordinarios son postulados al igual que los objetos más sofisticados de las teorías científicas más desarrolladas (PyO p. 36).

Aquí nos encontramos con una de las tesis más interesantes de Quine: la inseparabilidad del lenguaje y la teoría, entre conocimiento y significación. Esta inseparabilidad no refleja nuestra incapacidad subjetiva para distinguir el lenguaje y el conocimiento, es una inseparabilidad metafísica u ontológica, o por decirlo de otro modo, una inseparabilidad que se da 'en principio'.

“... nuestra comprensión de los objetos es en su mayor parte nuestro dominio de lo que la teoría dice acerca de ellos. No aprendemos primero el qué del discurso y luego el discurso sobre ello". (PyO p.29)

Los procesos de adquisición del lenguaje, son explicados por Quine conductísticamente a partir de la socialización. Pero los procesos de adquisición del lenguaje son inseparables de los procesos de conceptualización y de la construcción de los marcos referenciales desde los cuales hablamos acerca de objetos físicos corrientes, o de los objetos más llamativos de los que hablan las ciencias.

Estas consideraciones apoyan la idea, también sostenida por Quine, de que la semántica y la epistemología, aunque mantienen relaciones intrincadas e indirectas, son coextensivas.

${ }^{8}$ Cf. W. P., p. 223. 
Quine: Sobre referencia y relatividad - 125

"Aprender un lenguaje es aprender la significación de sus sentencias y, por lo tanto, aprender qué observaciones cuentan como evidencia favorable y como evidencia contraria a esas sentencias. La relación de evidencia y la relación semántica de la observación a la teoría son coextensivas". (RR p. 54)

Entonces es posible sostener que el estudio de los procesos de adquisición del lenguaje, puede llegar a dar cuenta también de los procesos de «adquisición» de teorías ${ }^{9}$. Las relaciones entre ambos aspectos ponen de manifiesto problemas semánticos por una parte y problemas epistemológicos por la otra. Los primeros hacen referencia a las cuestiones de significación y los segundos a las cuestiones del respaldo evidencial que toda ciencia necesita. Por esta razón también el análisis de las oraciones observacionales es la piedra de toque tanto en el tema de la traducción, en el aprendizaje del lenguaje como en el plano epistemológico.

El lenguaje es un «arte social» que se aprende mediante procedimientos de ensayo-error controlados socialmente, en relación a situaciones estimúlicas. Lo adquirimos con los únicos elementos de prueba de la conducta manifiesta de otros sujetos, bajo circunstancias públicamente reconocibles. Debido a esto puede ser estudiado empíricamente. Por su parte, la epistemología, en esta orientación naturalizada, podrá valerse de la estrategia genética utilizada para explicar los procesos de adquisición del lenguaje, para poder dar cuenta de las relaciones de soporte evidencial de los enunciados científicos. Es visible así que tanto el lenguaje como la teoría guardan relación con las oraciones observacionales.

${ }^{9} \mathrm{Cf}$. NNK, p. 74-76 y "Naturalización de la Epistemología" en La relatividad ontológica y otros ensayos, Madrid, Tecnos, 1974. (en adelante NE) 
"La sentencia de observación es básica para ambas empresas. Su relación... con nuestro conocimiento de lo que es verdadero, es, con mucho, la tradicional: las sentencias de observación son el receptáculo de evidencia para las hipótesis científicas. Su relación con el significado es también fundamental, puesto que son las que estamos en posición de aprender primero,... Ellas proporcionan el único acceso al lenguaje”. (NE, p. 117)

\section{Algunas consideraciones finales:}

Presentaremos brevemente algunas consideraciones sobre ciertas implicancias de las tesis de Quine desarrolladas en este trabajo.

En primer lugar, es necesario despejar algunas confusiones que han surgido de las distintas interpretaciones de la tesis de indeterminación de la traducción. Una de las más frecuentes ha sido la de entender que la tesis de indeterminación es una variante de la tesis de subdeterminación de las teorías empíricas.

Una versión de esta última tesis puede ser la siguiente:

-Las teorías sobre la naturaleza pueden ser lógicamente incompatibles entre sí y, sin embargo, ser empíricamente equivalentes en relación a la evidencia disponible.

Un manual de traducción -objetivo final de la tarea de traducción radical- ha de estar construido sobre la única base de los comportamientos lingüísticos observables y de procedimientos de tanteo interrogativo, de manera que desde el punto de vista metodológico, podría ser comparado con una teoría empírica. Las traducciones propuestas contarían como hipótesis, o conjuntos de 
ellas, que tendrán que ser sometidas a contrastación empírica por los métodos habituales. Pero la formulación de la tesis también dice que puede haber traducciones incompatibles entre sí pero compatibles con todas las disposiciones a la conducta verbal; y esto en el planteo de Quine significa que cualquier teoría está subdeterminada por todas las observaciones posibles. Ninguna cantidad de evidencia disponible garantizaría nunca que la traducción ofrecida fuera la única correcta. Pero esto no es todo. Además se dice no hay nada determinado en el significado de la oración original a lo que deban aproximarse las traducciones. Lo que significa en realidad la indeterminación, no es que no haya traducciones correctas, sino que siempre habrá más de una. Dos oraciones diferentes en un lenguaje pueden ser ambas traducciones correctas de una oración única en otro, y no hay nada objetivo que pueda dirimir la cuestión de cuál de las dos es la correcta. La traducción es indeterminada porque el significado es indeterminado.

La diferencia entre ambas tesis radica en que pertenecen a ámbitos de indagación diferentes. La tesis de subdeterminación es una tesis epistemológica, mientras que la tesis de indeterminación es una tesis ontológica. La subdeterminación se refiere al estatuto cognoscitivo de las teorías empíricas, y afecta a todo conocimiento humano. No hace más que reconocer la contingencia y la falibilidad de nuestro conocimiento del mundo. La indeterminación afecta a la naturaleza del significado -si se acepta el contrasentido-, nos dice que son los significados o mejor dicho, lo que no son. No son nada objetivo a lo cual se puedan aproximar las traducciones. No hay significados más allá de lo que pueda observarse en el comportamiento lingüístico.

Para un planteo de tradición analítica como el de Quine (con todas las distancias que lo separan de planteos semanticistas, por ej.: Frege, Russell, Carnap) la semántica guarda una relación 
estrecha con la ontología, ya que la primera es esencial para entender la relación del lenguaje con el mundo. Como hemos dicho, también, semántica y epistemología convergen en la filosofía quineana en aspectos básicos. Sin embargo, esto no quiere decir que se ocupen exactamente de las mismas cuestiones.

Ontología y epistemología se interesan por diferentes problemas. La ontología centra su atención en la cuestión 'acerca de lo que hay' (para ser fiel a la expresión quineana); y lo que hay es una cuestión acerca de la verdad. La epistemología centra su enfoque en cómo conocemos lo que hay; y esto -desde la perspectiva de la epistemología naturalizada- es una cuestión acerca del método y de la evidencia.

Como ya se ha dicho más arriba, lo que hay son los objetos postulados por las teorías, y en un sentido más general y naturalista, hay los objetos de los que hablamos en el lenguaje ordinario. La noción de hecho (fact of the matter) y la noción de verdad son nociones internas, pertenecientes a un marco referencial que es propio de cada teoría o de cada lenguaje.

"La importancia de esta noción (matter of fact) se muestra cuando la relacionamos, por ejemplo, con la doctrina de la indeterminación de la traducción. He sostenido que dos manuales de traducción en conflicto pueden ambos hacer justicia a todas las disposiciones para actuar, y que, en tal caso, no hay ninguin hecho de la cuestión (fact of the matter) de cuál de los dos manuales es el correcto. La noción propuesta de cuestión de hecho no es trascendental ni epistemológica, ni siquiera un asunto de evidencia; es ontológica y ha de tomarse de un modo naturalista dentro de nuestra teoría científica del mundo". (TyC.p. 34) 
La tesis de indeterminación de la traducción es una tesis controvertida, por lo que ha recibido interpretaciones que son diversas. También es una tesis importante, debido fundamentalmente a sus consecuencias para la reflexión filosófica. Representa, quizás, una de las aplicaciones más extremas del naturalismo en filosofía. Las constricciones que exige y la prohibiciones que impone, invitan a abandonar una serie de nociones y categorías vitales para el trabajo filosófico. Abandonar la noción de significado, es un asunto muy serio para la filosofía. En su momento, implicó una revisión muy profunda dentro del movimiento de la filosofía analítica.

Las influencias posteriores que ha tenido este "programa de investigación" son más que cuantiosas y, aunque un poco temerariamente, se podría afirmar que ha constituido un programa progresivo. Esta calificación no corre por cuenta solamente de los logros propios o los éxitos explicativos del mismo, sino, por sobre todo, por la líneas de investigación diversas cuyo desarrollo ha colaborado a estimular. Baste con mencionar el impulso dado a las orientaciones neurocientistas en epistemología, la marca profunda en la historia, la sociología y la filosofía de la ciencia poskuhniana, entre otros desarrollos.

La filosofía quineana en general y, en particular, las tesis analizadas en este trabajo, tienen la particularidad de plantear cuestiones que podrían denominarse 'metafilosóficas', entendiendo por ello una visión que considera inviable toda empresa filosófica concebida como independiente de la aceptación de ciertas creencias (compromisos) que son, ellos mismos injustificables, y por que no, hasta inescrutables. 


\section{REFERENCIAS BIBLIOGRÁFICAS:}

\section{Obras de W. V.QUINE}

PyO: Palabra y Objeto (1960). Barcelona, Labor, 1968.

R.O.: "Relatividad ontológica" en La relatividad ontológica y otros ensayos.(1969). Madrid, Tecnos, 1974.

N.E.: "Naturalización de la epistemología" en La relatividad ontológica y otros ensayos.

R.R.: Las raíces de la referencia.(1974). Madrid, Alianza, 1988.

W. P.: The Wats of Paradox. Harvard University Press, 1976.

Revised and enlarged edition.

TyC. "Las cosas y" su lugar en las teorías», en Teorías y cosas.(1981). México, UNAM, 1986.

B.V. La búsqueda de la verdad. (1990). Barcelona, Crítica, 1992.

\section{Otros:}

BENNETT, J. Locke, Berkeley, Hume: temas centrales. México, U.N.A.M., 1988.

GIBSON, R. "Translation, Physics and Facts of the matter" en The Philosophy of W.V. Quine. The Library of Living Philosophers. Volume XVIII. L. E. Hahn and P. A. Schilpp (eds.) Open Court, 1986.

ORAYEN, R. "Indeterminación de la traducción y epistemología naturalizada”, en Análisis Filosófico. Buenos Aires, SADAF, 1990. 